\title{
Memoria y percepción en la entrevista autobiográfica: una simulación episódica que se adapta en tiempo real al contexto*
}

\author{
Carlos Alberto Guerrero Velázquez \\ Universidad Autónoma del Estado de Morelos, Morelos, México \\ guerrerovelazquez@gmail.com \\ Recibido: 31 de octubre de 2020 | Aceptado: 09 de marzo de 2021 \\ https://doi.org/10.17533/udea.ef.n64a02
}

\begin{abstract}
Resumen: Normalmente se piensa en la percepción y la memoria como dos capacidades independientes, creyendo que la primera solo tiene influencia sobre la segunda durante la codificación. En las entrevistas autobiográficas de historia oral y memoria histórica, los entrevistados seleccionan, adaptan y completan sus recuerdos para crear diferentes versiones de ellos. En este artículo se argumenta que lo anterior es consecuencia de la naturaleza simulativa de la memoria episódica, y del empleo por los entrevistados de información perceptiva para generar y adaptar sus recuerdos a un discurso autobiográfico, buscando satisfacer un propósito comunicativo. Para ilustrar esto, se analizan tres factores contextuales que influyen sobre la construcción del recuerdo en una entrevista autobiográfica (objetivo comunicativo, idioma de comunicación e interacción emocional), mostrando que, en este tipo de recuperación, memoria y percepción contribuyen simultáneamente a construir simulaciones de eventos que se adaptan en tiempo real al contexto donde ocurre la evocación.

Palabras clave: entrevista autobiográfica, teoría de la simulación, memoria y percepción, memoria constructiva, recuerdo autobiográfico
\end{abstract}

* El presente artículo se basa parcialmente en la tesis "Factores contextuales que influyen en la evocación autobiográfica en un contexto de entrevista" (Guerrero Velázquez, 2020), cursada con el apoyo de una beca CONACYT, y aprobada con mención honorífica como trabajo final de la maestría en Ciencias Cognitivas en el Centro de Investigación en Ciencias Cognitivas (CINCCO) de la UAEM, en Cuernavaca, México. Agradezco profundamente por su guía y acompañamiento constante a los integrantes de mi comité tutoral: el Dr. Juan C. González González (tutor de tesis), el Dr. Gerardo Maldonado Paz y la Mtra. Susana Ramírez-Vizcaya. Así mismo, agradezco también al Dr. Kourken Michaelian por sus invaluables aportaciones para el desarrollo del proyecto, así como a los miembros del Centre de Philosophie de la Mémoire de la Université Grenoble Alpes por su retroalimentación y apoyo.

\section{Cómo citar este artículo:}

Guerrero-Velázquez, C. A. (2021). Memoria y percepción en la entrevista autobiográfica: una simulación episódica que se adapta en tiempo real al contexto. Estudios de Filosofía, 64, 21-45. https://doi. org/10.17533/udea.ef.n64a02

\section{OPEN ACCESS}

Estud.filos n. ${ }^{0}$ 64. Junio-diciembre de $2021 \mid$ pp. 21-45 | Universidad de Antioquia | ISSN 0121-3628 | ISSN-e 2256-358X 


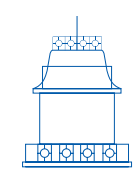

ARTÍCULO

DE INVESTIGACIÓN

\title{
Memory and perception in autobiographical interviews: an episodic simulation that adapts in real-time to the context
}

\begin{abstract}
Perception and memory are usually thought to be two independent faculties, where the former is believed to only have an influence on the latter at encoding. In autobiographical interviews of oral history and historical memory, interviewees select, adapt, and complete their memories to create different versions. This paper argues that this process is a consequence of the simulative nature of episodic memory and the interviewees' use of perceptual information to generate and adapt their memories to an autobiographical discourse with the goal of satisfying a communicative purpose. To illustrate this, three contextual factors (communicative aim, language of communication, and emotional interaction) that influence the construction of a memory in an autobiographical interview are analyzed, showing that, in this type of recall, memory and perception simultaneously contribute to constructing episodic simulations of events, which adapt in real-time to the context in which recall happens.
\end{abstract}

Keywords: autobiographical interview, simulation theory, memory and perception, constructive memory, autobiographical remembering

\section{Carlos Alberto Guerrero Velázquez}

es Licenciado en Filosofía (UAA - México), Especialista en Ciencias Sociales con Mención en Historia Social (UNLu - Argentina), y Maestro en Ciencias Cognitivas (UAEM - México). Sus intereses de investigación giran alrededor de los temas de identidad, memoria, lenguaje, mente y cognición, tendiendo siempre al enfoque interdisciplinar y al encuentro entre la investigación empírica y la reflexión filosófica.

ORCID: 0000-0002-1384-7601 
A mi madre Leticia y mi padre Alberto, que son y serán siempre la más valiosa y amada de mis memorias.

\section{Introducción}

Los atentados terroristas del 11 de septiembre de 2001 que destruyeron el World Trade Center de Nueva York (conocidos popularmente como 11-S) fueron un capítulo importante de la historia contemporánea y la memoria histórica del pueblo estadounidense. Una parte del suceso pudo ser seguido en tiempo real y a nivel internacional por la cobertura televisiva que se hizo de él. Siete semanas después del siniestro, Pezdek (2003) realizó un estudio en el que participaron estudiantes universitarios, cerca de la mitad de ellos residentes de Manhattan (lugar del ataque). En dicho estudio se hicieron entrevistas autobiográficas (aquellas que tienen por objeto conocer los recuerdos de una persona sobre uno o varios episodios de su vida) con el fin de analizar los recuerdos de los participantes con relación al 11-S. Una de las preguntas realizadas fue: "El 11 de septiembre, ¿vio en la televisión el video del primer avión [secuestrado] golpeando la primera torre?". El 73\% de los participantes contestó que sí, a pesar de que eso no era posible, debido a que las filmaciones de la primera nave impactando el WTC no se hicieron públicas sino hasta un día después de los sucesos.

El anterior es un claro ejemplo de uno de los fenómenos más comunes en las entrevistas autobiográficas: al evocar un suceso, las personas comunican sus recuerdos elaborando versiones variadas de ellos a partir de la incorporación u omisión de detalles y características pertenecientes a otros episodios o a información relacionada con el incidente. En las entrevistas también es común que, además de recordar, los entrevistados realicen otros procesos cognitivos como evaluar comportamientos o pensar de modo contrafáctico (Arfuch, 1995; Grele, 1989; Portelli, 2017). Todo lo anterior se conoce en estas disciplinas sociales como "carácter creativo del recuerdo" (Arfuch, 1995; 2007) y pone de relieve el talante al menos parcialmente ficcional del discurso episódico que se genera en las entrevistas autobiográficas. En estas, el proceso de evocar se lleva a cabo en una interacción dialógica (en conversación con el entrevistador) y muchos detalles de la elaboración del recuerdo se hacen públicos, permitiendo a un observador externo hacer un seguimiento del curso de la evocación y de la forma en que se construye en tiempo real el discurso autobiográfico.

El carácter creativo del recuerdo ha sido de gran interés para los teóricos de la entrevista autobiográfica (Arfuch, 1995; Grele, 1989; Robin, 1996), y supone problemas epistemológicos y metodológicos que no han sido aún tratados con suficiencia; como la causa de la diferencia entre episodio vivido y recordado, el papel activo o pasivo de la memoria durante la recuperación, y la influencia de las aferencias interoceptivas y exteroceptivas durante la evocación. Entre los teóricos de la entrevista social, este 
fenómeno ha sido explicado principalmente como una consecuencia de la interacción dialógica que se da entre entrevistado y entrevistador, aunque no es común, sin embargo, que historiadores orales e historiadores de memoria histórica (Cohen, 2006; Grele, 1991; Portelli, 2017), profundicen en los procesos psicológicos o neurológicos que subyacen a la evocación y los factores que la afectan-debido principalmente a que su enfoque de estudio y su nivel explicativo es otro. Tales procesos han sido estudiados más bien por psicólogos y filósofos de la memoria (Conway, 2005; 2015; Michaelian, 2016; Michaelian \& Sant'Anna, 2019; Schacter, 2003), aunque su análisis se ha enfocado comúnmente en la evocación en general y no en la evocación autobiográfica en un contexto de entrevista. Además, estos análisis, en el caso de los psicólogos, comúnmente se centran en la actividad mental del individuo (más específicamente, intracraneal) y dejan de lado los factores externos o contextuales que puedan estar influyendo en el proceso, debido principalmente, a que el diseño de un paradigma experimental exige seleccionar - de entre todas las variables posiblesaquellas que puedan ser controladas y medidas con mayor precisión. En psicología, por ejemplo, la variación entre episodio vivido y recordado ha sido explicada como un efecto provocado por factores como el paso del tiempo, la atribución errónea de la fuente, los sesgos retrospectivos que se dan al recordar (Shacter, 2003); la necesidad de que nuestros recuerdos sean coherentes con la identidad personal o de que sirvan para explicar una situación actual (Conway, 2005).

En este artículo se analiza y describe, desde una perspectiva cognitiva que integra distintos niveles de explicación, el proceso de evocación que se lleva a cabo en la entrevista autobiográfica, con fundamento en literatura de psicología, neuropsicología y filosofía de la memoria. Se pretende mostrar que el carácter creativo del recuerdo autobiográfico en un contexto de entrevista se produce debido a que, al recordar, la memoria humana emplea contenido episódico y semántico para construir versiones del recuerdo que se pretende evocar (Michaelian, 2016; Michaelian \& Santa Anna, 2019), y además incorpora información perceptiva interoceptiva y exteroceptiva para dirigir, enriquecer y retroalimentar esa construcción.

Para lograr tal propósito, primero se abundará en la entrevista autobiográfica para describir lo que los historiadores han señalado como el carácter creativo del recuerdo. Posteriormente se abordará la evocación dirigida que se da en un contexto de entrevista, buscando analizar los procesos cognitivos implicados en ella desde las teorías de simulación mnémica (Michaelian, 2016; Schacter, 2018). A continuación se explicará la influencia que la simulación episódica tiene en la construcción de los recuerdos y su relación con el carácter creativo de la evocación autobiográfica. Finalmente, con el propósito de ilustrar cómo es que la memoria emplea información perceptiva para la construcción de la simulación, se describirán tres factores contextuales que influyen sobre ella en el contexto de una entrevista autobiográfica (objetivo comunicativo, idioma de comunicación e interacción emocional). Se mostrará, con lo anterior, que en este tipo de evocación, la memoria y percepción 
trabajan simultáneamente para elaborar versiones de los eventos que se adaptan en tiempo real a las necesidades del contexto.

\section{I - La entrevista autobiográfica y el carácter creativo del recuerdo}

La entrevista autobiográfica' es el suceso comunicativo que se da a partir de la interacción dialógica entre un entrevistador y un entrevistado, el cual es una persona que evoca recuerdos sobre uno o más sucesos de su vida y los organiza en una narración con sentido, llamada "discurso autobiográfico"2 (Arfuch, 1995; 2007). Se puede considerar un dispositivo epistémico (siguiendo a Fisher, 2006) en tanto que permite a los participantes recordar conocimiento biográfico -tanto episódico como semántico-, organizarlo y crear nuevos contenidos a partir de él.

En las disciplinas de la historia oral y los estudios de memoria histórica se ha señalado en varias ocasiones el carácter creativo del discurso autobiográfico producido en un contexto de entrevista (Barela \& García, 2009; Cohen, 2006; Grele, 1989; Pollack \& Heinich, 2006; Portelli, 2017), debido principalmente a dos razones. La primera es que no solamente se narran sucesos evocados, sino que se expresan distintas dimensiones de la identidad de una persona, como valores, significación del pasado, sentido histórico, objetivos personales, propósito comunicativo, entre muchos otros (Arfuch, 1995; De Garay-Arellano, 1999; Régine, 1996); para los fines de este trabajo, se nombra a esta característica la expresión del yo [EdY]. La segunda razón es que todos estos factores guían de una u otra forma la organización del discurso, la selectividad de los recuerdos a comunicar y su ordenamiento dentro de una narración coherente, en la cual se mezclan la evocación del episodio con otros procesos cognitivos que la persona realiza al momento de recordar, como evaluar acciones propias o ajenas, considerar comportamientos alternativos de acción (pensamiento contrafactual), reflexionar sobre las consecuencias de la actuación, entre muchos otros; característica que en este texto se denomina realización de procesos cognitivos alternos [RPCA]: al parecer, las personas tienden a emplear sus recuerdos de un episodio para, además de recordar, construir sentidos relacionados con el suceso o su actuación en este.

Otro aspecto importante de la evocación autobiográfica que se da en contexto de entrevista (y que será profundizado en la tercera sección de este trabajo) es que un entrevistador la guía. Esto supone una creación dialógica del discurso autobiográfico (Arfuch, 1995; 2007), pues la construcción del recuerdo se da en interacción con el otro y no en una vivencia personal privada, como sucede en la evocación espontánea.

1 Pueden verse ejemplos de este tipo de entrevistas y su método en Arfuch (1995; 2007); Actis, Aldini, Gardella, Lewin \& Tokar (2006), De Garay-Arellano (1999), Barela, Miguez \& García (2009).

2 Llamado también "autobiografía" o "narración de vida" en la historia oral. En este trabajo se define como la expresión pública lingüística del recuerdo autobiográfico que se construye en un contexto de entrevista, ya sea de un solo recuerdo o de varios. 
En la entrevista, la evocación y la elaboración del discurso autobiográfico se ven influenciados por la presencia del entrevistador, la idiosincrasia de este, las expectativas que fija sobre el entrevistado, su propia comprensión o su necesidad de mayor detalle. Por ejemplo, en una entrevista que se haga a un integrante de una organización política conocido por su militancia, aunque las preguntas no se hagan directamente con respecto a tal militancia, el aspecto político permeará muchas de las respuestas, las cuales serán interpretadas de cierta manera por las opiniones políticas que tenga el entrevistador; este elaborará o reorientará sus cuestionamientos a partir de tales opiniones, de la claridad que perciba en el relato, del motivo de la entrevista o de los detalles que requiera para enriquecer o comprender el episodio (Greele, 1989). Lo anterior provocará que el entrevistado oriente y acomode su discurso (amplíe, omita, enfatice o reelabore detalles o ideas de su narración) para participar en tal interacción, satisfacer los requisitos que plantea el entrevistador, y comunicar lo que desea.

A pesar del énfasis que se ha puesto en el carácter creativo y al menos parcialmente ficcional del discurso autobiográfico y de las implicaciones de la interacción dialógica en la entrevista, los teóricos de esta (Arfuch, 1995; Robin, 1996), así como de la literatura en general sobre el método de la historia oral o memoria histórica (como puede verse en Barela, Miguez \& García, 2009; De Garay-Arellano, 1999; GonzálezMonteagudo, 2010; Greele, 1989) no profundizan en los procesos psicológicos que se dan en este tipo de evocación, ni en por qué el recuerdo autobiográfico tiene un carácter constructivo. Las teorías de simulación mnémica pueden servir como un recurso que permite describir - desde el punto de vista cognitivo- de qué forma se da la evocación autobiográfica en contexto de entrevista, así como señalar las características que la hacen adquirir tal carácter creativo.

\section{II - El recuerdo como simulación episódica}

Hablar de evocación autobiográfica exige primero aclarar en qué consiste el proceso cognitivo de evocación. Se empleará el postulado teórico de las teorías de simulación mnémica por considerarlo un recurso metodológico robusto -y cada vez más sustentado en evidencia experimental- para explicar los distintos fenómenos de la memoria, ${ }^{3}$ encontrando acogida tanto en filósofos de la memoria (De Brigard, 2014;

3 Existen distintos postulados teóricos sobre memoria que pueden emplearse para cumplir con el propósito de este análisis, ya que actualmente existe un consenso generalizado sobre el carácter constructivo de la memoria humana (véase Michaelian \& Sant’Anna, 2019 para una revisión de tales teorías). Para este trabajo se ha elegido la teoría de la simulación mnémica por distintas razones. En primera, por ser un postulado que goza de una aceptación multidisciplinaria. Lo anterior, como segunda razón, conlleva que se haya desarrollado abundante argumentación teórica e investigación empírica en torno a ella (véase Schacter, 2018). En tercera, a diferencia de otros postulados teóricos -como la teoría causal de la memoria 
Michaelian, 2016; Michaelian \& Sant'Anna, 2019; Shanton \& Goldman, 2010), como en psicólogos y neuropsicólogos (Barsalou, 2005; Cheng, Werning \& Suddendorf, 2016; Schacter, 2018).

Según Michaelian (2016), al evocar algo los seres humanos empleamos experiencias pasadas relacionadas al suceso que deseamos recordar y las combinamos de forma flexible para generar simulaciones que nos permiten imaginar ${ }^{4}$ un episodio; el producto de esta simulación es lo que experimentamos como recuerdo, lo que en otras palabras significa que recordar el pasado consiste en imaginarlo. Este postulado se ha visto reforzado por evidencia empírica que muestra que existe un traslape en las redes neuronales que empleamos para recordar un episodio y para imaginar el futuro u otras situaciones probables (Schacter, 2018). Tanto Michaelian (2016) como Schacter (2007; 2018) coinciden en señalar que tales procesos son realizados por un mismo sistema cuya función, en general, es realizar simulaciones episódicas. Para Schacter (2018), el propósito evolutivo de este sistema, más que recordar, es habilitarnos para emplear experiencias pasadas y usarlas en la simulación de futuros probables, permitiéndonos así calcular con antelación las implicaciones y consecuencias de una posible situación futura. Michaelian (2016) se refiere a este como "sistema de construcción episódica" (SCE, en lo sucesivo) y señala que se trata de un sistema de construcción capaz de realizar distintos procesos (imaginar el futuro, planear acciones, imaginar escenas ficticias, entre otros), siendo la evocación solo uno más de ellos. ${ }^{5}$

Para Schacter (2018), la habilidad de simular experiencias futuras y construir representaciones de eventos, puede contribuir a errores de la memoria, lo que significa que las desviaciones, omisiones, falsas atribuciones y otros efectos que se dan en la evocación autobiográfica, ${ }^{6}$ pueden ser una consecuencia de las características propias del sistema que posibilita la simulación y de que su propósito adaptativo no se agote solamente en recordar. Pero esta falibilidad de la memoria en realidad significa una ventaja adaptativa, ya que "no necesitamos registrar cada detalle de cada experiencia,

(véase Michaelian y Robins, 2018)-, la teoría de la simulación otorga un rol principal a la imaginación en la construcción del recuerdo. Esto resulta de gran importancia si se considera que la imaginación tiene un papel fundamental en fenómenos de la memoria como la existencia de falsos recuerdos, la ocurrencia de procesos cognitivos alternos durante la evocación o la posibilidad de emplear un recuerdo como un dispositivo epistémico (algunos se explicarán a lo largo del texto).

4 Existe un gran debate sobre en qué consiste la imaginación. El uso que se le da en este texto es como sinónimo de imaginería mental, empleando la definición de Moulton \& Kosslyn (2009), para quienes la imaginería es un proceso cognitivo que consiste en la simulación mental de una situación; simulación que se emplea para hacer explícitas y accesibles las consecuencias de una acción.

5 Para Michaelian (2016), existe una distinción entre la memoria episódica, que nos habilita para obtener conocimiento sobre episodios experimentados en el pasado, y la autobiográfica, que no se relaciona con un sistema específico de memoria, sino que emerge de la interacción de otras capacidades más fundamentales, incluyendo la memoria episódica y semántica. En este sentido, la evocación autobiográfica emplea el sistema de construcción episódica para imaginar un episodio pasado haciendo uso tanto de conocimiento episódico como semántico, provocando que se manifiesten diferentes dimensiones de la identidad de una persona al imaginar un recuerdo autobiográfico.

6 Shacter $(1999 ; 2002)$ estudia estos efectos y los describe ampliamente en su obra The seven sins of memory. 
sino extraer las características centrales, el significado o la esencia de las experiencias pasadas, lo cual es fundamental para funciones tan importantes como nuestra capacidad para categorizar y generalizar" (Shacter, 2018, p. 6). De acuerdo a los teóricos de la simulación, a diferencia de otras especies animales, la memoria humana ha evolucionado para ser capaz de integrar conocimiento de distintas experiencias y combinarlo para simular las situaciones requeridas. Esta característica evita, entre otras cosas, el enorme gasto de energía que supondría registrar cada episodio vivido de forma detallada y después reconstruirlo o representarlo mentalmente con todo detalle. En otras palabras, el que la memoria humana sea constructiva y no reproductiva significa una enorme ventaja adaptativa.

¿De qué forma se construye una simulación episódica? De acuerdo con Michaelian (2016), la construcción que se realiza en el SCE emplea de forma flexible, junto con conocimiento semántico e información contextual, experiencias de distintos eventos que están codificadas en las huellas de memoria. ${ }^{7}$ Siguiendo a De Brigard (2014), estas huellas pueden entenderse como propiedades disposicionales de las redes neuronales a provocar ciertas respuestas. Durante la codificación (también llamada "registro" de la memoria), se da el fortalecimiento de conexiones neuronales debido a la co-activación de diferentes regiones del cerebro. Una huella de memoria es la propiedad disposicional que estas regiones tienen a la reactivación, cuando son estimuladas por la señal correcta, en aproximadamente el mismo patrón de activación que experimentaron durante la codificación (De Brigard, 2014). El SCE emplea, entonces, contenido ${ }^{8}$ de múltiples experiencias habilitado por estas huellas de forma flexible para construir una versión del episodio que se desea recordar, permitiendo con ello que imaginemos el recuerdo en cuestión.

Las teorías de simulación mnémica -especialmente el concepto de SCE como lo plantea Michaelian (2016) y que se ha delineado en esta sección- permiten analizar la evocación que se da en las entrevistas autobiográficas y explorar sus particularidades, siendo el carácter creativo del recuerdo autobiográfico la más importante en este trabajo. Como se mencionó en la sección anterior, este carácter se ve reflejado en dos características descritas por los teóricos de la entrevista: la realización de procesos cognitivos alternos (RPCA) y la expresión del yo (EdY).

La RPCA es muy común durante la evocación autobiográfica: las personas entrevistadas tienden a emplear sus recuerdos para distintas tareas, como la

7 Las huellas empleadas no necesariamente se originan en el evento que se pretende recordar, por lo que no necesariamente existe una conexión causal entre la experiencia particular vivida y el recuerdo.

8 "Contenido" no debe entenderse como algún tipo de objeto mental que se encuentra almacenado en el cerebro, lo que corresponde al entendido tradicional de "contenido semántico", sino a la representación que se construye a partir de la activación de las redes neuronales que constituyen las huellas de memoria. En este caso, el contenido no existe previo a la activación, sino a partir de esta, y es un componente constitutivo del proceso de recordar, no el recuerdo en sí (entendido como contenido almacenado previamente). Como señalan Michaelian \& Sant’Anna (2019), la evocación episódica puede verse como un proceso que involucra la transición de un estado sin contenido (contentless) a uno con contenido (contentfull). 
actualización de significados a partir de la reconstrucción del suceso ("ahora que lo pienso, eso que pasó me ayudó a..."), el pensamiento condicional contrafáctico ("¿y qué habría ocurrido si...?"), la auto-corrección de información ("ahora que lo pienso, no pudo haber sido así, porque en ese momento yo estaba..."), la incorporación de detalles externos a la vivencia de la experiencia ("dieron el golpe cuando yo estaba incomunicado..."), entre muchos otros. Estos procesos son distintos entre sí, pero tienen en común el estar basados en la imaginación de un episodio y en que esa imaginación es empleada para realizar distintas tareas que, en un momento dado, nos pueden servir para imaginar escenarios posibles o ensayar consecuencias de la actuación. Se puede considerar que la RPCA que se da durante la evocación autobiográfica es una consecuencia natural del funcionamiento del SCE, pues el contenido ${ }^{9}$ de la imaginación episódica, aunque haya sido integrado para recordar, permite (e invita a) que se realicen distintos procesos, combinándose de formas variables para generar predicciones, intenciones, planificación, etc. (Szpunar, Spreng \& Schacter, 2014). En otras palabras, los seres humanos realizamos otros procesos cognitivos alternos al momento de recordar debido a dos razones: la primera, que todos esos procesos son habilitados por el mismo sistema; y la segunda, que el propósito evolutivo del mismo, más que recordar, es permitirnos imaginar situaciones posibles, razón por la cual empleamos el contenido evocado para ello.

La segunda característica del carácter creativo del recuerdo, la EdY, se refleja en la expresión de múltiples dimensiones de la identidad de una persona que se da durante el recuerdo autobiográfico, como la enunciación de valores personales y grupales, la significación del pasado, la atribución de sentido histórico al suceso, la proyección o construcción que se hace de sí mismo en el episodio, el rol que juega el sujeto en las acciones, etc. Como se ha visto, para generar una simulación, el SCE combina de forma flexible distintas experiencias pasadas, las cuales pueden ser de muy diversos tipos, dependiendo de lo que se pretende recordar; estas experiencias están codificadas en las huellas de memoria y su activación depende de la señal que actúa como disparador. La ocurrencia de la EdY muestra que esta combinación no se limita solamente a experiencias de tipo episódico, sino que la construcción de la simulación se ve afectada por el conocimiento personal (llamada por Conway [2005] la "base de datos del yo") y que la imaginación del pasado tiende a construirse de forma coherente con la identidad personal. ${ }^{10} \mathrm{~A}$ partir de lo expuesto más arriba y siguiendo la teoría de la simulación, es posible suponer que la ocurrencia de la EdY se debe, por un lado, a que las huellas de memoria activadas durante la evocación no se limitan a experiencias sino también al conocimiento personal; y por otro, a que el recuerdo tiende

9 Entiéndase "contenido" como el producto de la imaginación episódica, es decir, lo representado en esa imaginación o aquello que se imagina.

10 Conway $(2001 ; 2005)$ señala que las evocaciones autobiográficas emplean conocimiento sobre la historia e imagen personal de quien recuerda, privilegiando la información referida a sí mismo para generar el recuerdo. 
a construirse de forma coherente con la identidad, reforzando así la imagen propia y evitando la disonancia cognitiva (Conway, 2005). Es debido a ello que en los discursos autobiográficos realizados en contexto de entrevista, junto con el recuerdo, a menudo se expresan valores personales ("lo hice a pesar de saber que no era correcto"); valores grupales ("no podía dejar atrás a los compañeros"); asignación de un rol en la historia ("yo siempre fui el tímido de la familia"); imagen de sí mismo ante el suceso ("ahora veo que yo era muy vulnerable entonces"); justificación de las acciones ("me atreví a matar porque era necesario, pero yo no soy un asesino"); entre muchas otras.

Un ejemplo de lo anterior puede verse en la síntesis evaluativa y la proyección de la imagen personal que se hace presente en el discurso autobiográfico de una mujer superviviente de detención ilegal y tortura, que fue recluida en el centro clandestino de la ESMA (Escuela Superior de la Armada) durante la última dictadura argentina. Durante una entrevista autobiográfica donde se le preguntó por momentos de su vida en el centro, mientras narraba episodios de su experiencia, expresó: "Ahora me doy cuenta de que yo, estando adentro, sentía como si me hubieran puesto un vidrio que me separaba del mundo. Sabía que mi nombre no tenía el mismo valor que antes, era un nombre desaparecido" (Gardella, como se cita en Actis et al., 2006). En otros casos se integran conocimientos históricos, sociales o políticos al discurso autobiográfico para complementar y dotar de sentido al recuerdo, pero también para brindar una referencia histórica que ayude a entender el pasado y la forma en que la persona se ve afectada por él. Por ejemplo, en una entrevista realizada a la hija de un militante desaparecido durante la última dictadura argentina, cuando se le preguntó por un recuerdo de sus padres, ella señaló: "Mis padres tenían una vida militante importante. Yo soy de San Salvador de Jujuy y en el año 74 después de que muere Perón, se la llevan detenida a mi mamá, en Jujuy. Ahí deciden que veníamos a Buenos Aires" (Arroyo, citada por Cueto, 2008).

Un último aspecto a considerar en esta parte es la integración de conocimiento semántico y episódico de múltiples recuerdos que se da a menudo en la evocación autobiográfica, y que los historiadores ven reflejada en la construcción del discurso en contexto de entrevista. En el estudio citado al inicio de este texto (Pezdeck, 2003), una gran parte de los entrevistados sobre el atentado del 11 de septiembre al WTC recordó haber visto la imagen del primer avión secuestrado impactando una de las torres, durante la transmisión en vivo del siniestro. Resulta evidente que las imágenes transmitidas televisivamente en días posteriores, a pesar de que no correspondían temporalmente a lo observado el día del siniestro, fueron evocadas también como parte del recuerdo porque forman parte del conocimiento del episodio. Lo mismo sucede en otros casos, donde las personas completan sus recuerdos con información sobre el suceso, detalles de los espacios donde este sucede, y muchos otros datos aprendidos antes o después del episodio. Lo anterior puede obedecer a dos razones. La primera es la forma en que funciona el SCE, y que se ha descrito con anterioridad: al imaginar el episodio, se emplean experiencias pasadas de manera 
flexible para generar la simulación, lo cual supone que el episodio imaginado a menudo contenga detalles que podrían corresponder a otros episodios o a información sobre el suceso. La segunda razón es que el propósito adaptativo de la memoria humana es permitirnos imaginar escenarios posibles y calcular las consecuencias de acciones futuras (Schacter, 2018): en términos de supervivencia, es más importante ser capaces de emplear la información más saliente de múltiples experiencias y generar así escenarios de actuación más detallados, que recordar de forma puntual cada detalle de una sola experiencia. En otras palabras, las personas integramos conocimiento de distintas experiencias al recordar porque eso no permite construir simulaciones más detalladas, lo que nos vuelve mejores para predecir."

Esto último abre también la puerta para pensar en otra característica del recuerdo episódico, que es el empleo de información contextual en la construcción de la simulación. La idea de Schacter (2018) de que el propósito de nuestra memoria episódica es imaginar situaciones posibles para predecir consecuencias, conlleva pensar en una memoria motivada (guiada por objetivos) cuyos procesos se llevan a cabo para ayudarnos a mejorar nuestras posibilidades de actuación y éxito en el presente y futuro. Son los estímulos actuales, tanto contextuales como reflexivos, los que activan, motivan y guían nuestras imaginaciones episódicas. En este sentido, resulta lógico pensar que el contenido empleado para la construcción de una simulación no se limita solamente a nuestras experiencias pasadas, sino que incluye también información perceptiva que le permite al agente obtener información de su contexto. Se profundizará en ello en la tercera parte de este artículo.

\section{III - Factores contextuales que influyen en la construcción del recuerdo}

Como se mencionó anteriormente, existen distintos factores que influyen en la evocación autobiográfica. En esta parte se tratan aquellos que surgen debido a la situación contextual de la persona que evoca y la interacción comunicativa dinámica que se da entre los hablantes (entrevistador y entrevistado[s]). ${ }^{12}$

11 La habilidad de la memoria humana para hacer abstracciones y generalizaciones en lugar de registros precisos puede verse también en otros tipos de memoria. Experimentos que emplean el paradigma Deese-Roediger-Mcdermott (DRM) (Deese, 1959; Roediger \& Mcdermott, 1995) muestran que si se presenta una lista de palabras semánticamente relacionadas a una persona ("gato, oso, león, jirafa, mamíferos"), esta tenderá a recordar falsamente una palabra señuelo no incluida en la lista ("animales"). Lo anterior es un ejemplo de que, más que recordar información puntual o detalles específicos, nuestra memoria -en su funcionamiento normal- es mucho más hábil en recordar de una forma temática y global. Esto puede significar una gran ventaja adaptativa en términos de reducción del gasto energético empleado en el proceso cognitivo del recuerdo y de optimización para la construcción de escenarios probables, si seguimos a Schacter (2018).

12 Aunque el propósito de este texto no es abundar en la discusión sobre la naturaleza del lenguaje, es importante recordar la distinción que existe entre el mismo y la comunicación, ya que en distintos animales no humanos se observan sistemas de 
Durante la evocación autobiográfica en una entrevista la simulación mnémica se construye y adapta para satisfacer un propósito comunicativo, por lo que el entrevistado incorpora información perceptual contextual que influye en tiempo real sobre la imaginación del episodio. En una entrevista, el entrevistado percibe, decodifica e interpreta estímulos perceptivos visuales, auditivos y táctiles producidos por el entrevistador u otras personas, y se vale de ellos para fijar el objetivo de la indagación mnémica (lo que se le pide que recuerde), conocer las condiciones y detalles que debe tener el reporte del recuerdo, o recibir retroalimentación sobre la calidad de su relato. Esta información le permite también adaptar su construcción episódica "sobre la marcha", elaborando versiones del episodio evocado que se modifican dependiendo de la recepción de su discurso. Los factores que se exponen a continuación muestran que, en la evocación autobiográfica producida durante una entrevista, la memoria se vale de la información perceptiva del contexto para realizar construcciones episódicas que satisfagan las necesidades de actuación del agente.

En la entrevista autobiográfica es especialmente notoria la relación que existe entre percepción y memoria durante la construcción del recuerdo episódico, ya que este tipo de evocación requiere necesariamente la interacción dinámica del agente con su contexto y con los sujetos que forman parte de él. Tal vez podría discutirse si los estímulos perceptivos tienen influencia en la evocación espontánea privada, ${ }^{13}$ pero en una situación conversacional (como lo es la entrevista) esta relación se vuelve mucho más evidente porque la interacción con el contexto forma parte constitutiva del proceso de recordar: el estímulo que detona la evocación es externo (pues la pregunta que fija el objetivo de la evocación proviene del entrevistador); la construcción del recuerdo se hace de forma lingüística (empleando el lenguaje oral para narrar, describir, explicar o detallar el evento) y se constriñe a las particularidades del idioma empleado por los interlocutores o las formas de expresión habituales (como las estructura narrativa o el uso de jergas); el entrevistado interpreta el lenguaje verbal y corporal del entrevistador y/o de otros entrevistados porque sabe que su relato está siendo evaluado, etc. Estas características, entre muchas otras, nos muestran la dependencia que tiene la memoria de la percepción para la consecución exitosa del(los) propósito(s) del agente. ${ }^{14}$

comunicación sofisticados que, sin embargo, no cumplen con ciertas características que sí tienen los lenguajes humanos. Esta distinción es imprescindible para comprender que aunque el lenguaje es una forma de comunicación, no toda forma de comunicación es un lenguaje (véase Smith \& Kosslyn, 2008). En este texto se guarda esta distinción empleando el término "comunicación" como referencia a un intercambio de información (puede ser no verbal), y "lenguaje" cuando se habla de un sistema de comunicación humano que cuenta con un sistema de signos y tiene características específicas (dualidad de patrón, arbitrariedad, capacidad generativa, recurrencia).

13 Aunque si se comparte una visión corporizada, situada o extendida de la cognición, es muy poco probable que se discuta este punto (véase Barsalou, 2008; Fuchs, 2012; Rietveld, Denys \& Van Westen, 2018).

14 Algunos ejemplos de los objetivos que se presentan en la entrevista pueden ser: comunicar, convencer, despertar emociones, defender su actuación, congraciarse, verse aceptado, mostrarse con un rol en la historia, mover a la acción, entre muchos otros. 
Los factores que se exponen en esta sección se detectaron al analizar las características de la evocación autobiográfica en entrevista, tanto las mencionadas en el párrafo anterior como otras. Los mismos se exponen con el propósito de ilustrar la forma en que la construcción episódica es llevada a cabo por el SCE y cómo se ve influenciada por información perceptiva tanto interoceptiva como exteroceptiva. Tales factores, aunque han sido explorados en distintas investigaciones (las cuales se mencionan a lo largo de esta sección), no se han enfocado en la habilidad específica de la evocación autobiográfica. Debido a las consideraciones de espacio, en este artículo se describen solamente los 3 más representativos del efecto mencionado (objetivo comunicativo, idioma de comunicación e interacción emocional), en lugar de los 6 que se analizaron en la investigación original que sirvió como base para la elaboración de este texto, ${ }^{15}$ en espera de abordar los restantes en otra oportunidad.

\section{A) Objetivo comunicativo:}

La simulación episódica se construye a partir del contenido habilitado por las huellas de memoria. La activación y selección de tal contenido es guiada por el objetivo comunicativo, que se establece mediante la interacción dialógica.

Como ya se ha descrito, el estímulo que desata la construcción del recuerdo en un contexto de entrevista es la pregunta del entrevistador, la cual es interpretada por el entrevistado para obtener información acerca de lo que se espera de su reporte mnémico. Esta pregunta provoca que la activación de huellas de memoria relacionadas con el suceso (Michaelian \& Sant'Anna, 2019) sea selectiva y esté orientada por un objetivo fijado lingüísticamente, a diferencia de la evocación espontánea. Supongamos que un entrevistador pregunta: “¿cuál fue tu reacción al darte cuenta de que el edificio podía caerse durante el sismo?". Esta pregunta ocasionará que se active en el entrevistado conocimiento relativo al suceso, como recuerdos episódicos sobre el sismo en cuestión, información del suceso leída o escuchada en medios de comunicación, conocimiento sobre las reacciones posibles de una persona en situaciones de riesgo, posibles consecuencias de un derrumbe, información sobre su imagen personal (si se considera a sí mismo valiente, arriesgado, temerario, etc.), entre muchos otros. También se activará conocimiento que no es relevante para la indagación y que no será empleado, información que no se desea comunicar, detalles que se descartarán por considerarlos nimios, etc.

15 La mencionada investigación fue un trabajo teórico de tesis de maestría en ciencias cognitivas, desarrollado a lo largo de 18 meses. Los seis factores que se analizaron se dividieron en dos secciones, dependiendo de su relación con otras habilidades cognitivas además de la percepción. Un primer grupo lo constituyeron los factores relacionados con el lenguaje: objetivo comunicativo, idioma de interacción y estructura narrativa. El segundo grupo lo formaron aquellos relacionados con las emociones: estado de ánimo, interacción emocional y presión social. 
A pesar de disponer de una gran cantidad de contenido, no todo es empleado para imaginar el recuerdo, pues no resulta relevante para el objetivo que se ha planteado. El acto de recordar, en este caso, se asemeja a una búsqueda: tratamos de encontrar los elementos que mejor nos ayuden a construir la escena, conscientes de que nuestro interlocutor espera obtener información de nuestro recuerdo. La interacción lingüística permite, así, que el entrevistador comunique sus expectativas sobre el tipo de recuerdo esperado y la forma en que el discurso debe orientarse; con ello define características del recuerdo como el papel de la persona entrevistada en el suceso que se está reconstruyendo (si este se proyecta como líder, agente de los hechos, observador de los mismos o algún otro personaje dentro de la narración); el objetivo de la indagación (le indica al entrevistado qué se está buscando en el recuerdo); o las características narrativas del recuerdo (si se debe dar una descripción, conducir a una evaluación del suceso, hacer una introspección, etc.). Esta expectativa permite que, a partir del abundante contenido habilitado por la activación de las huellas de memoria, la persona seleccione los elementos que deben formar parte de su narración y que construya la simulación episódica del acontecimiento a partir de esos elementos.

Pero el objetivo comunicativo no solamente se fija al principio de la indagación, sino que se adapta dinámicamente a las necesidades comunicativas que se actualizan, conforme la evocación avanza, mediante la interacción entre los hablantes. Mientras elabora su discurso autobiográfico, el entrevistado recibe y procesa información perceptual, en este caso lingüística, que le permite evaluar el éxito de su comunicación (definido por el nivel de logro planteado) y confirmar, refinar o modificar su objetivo (el motivo que guía la búsqueda) y su estrategia comunicativa (si hay que ampliar, ocultar, enfatizar algún punto, detallar o modificar el relato). Lo anterior ocasiona que el sujeto incorpore en su construcción episódica el contenido necesario para lograr su intención, imaginando una versión del recuerdo que pueda satisfacer la expectativa. Es por ello que los entrevistados pueden hacer un discurso autobiográfico distinto de un mismo suceso si el entrevistador, por ejemplo, expresa que el fin de la entrevista es conocer a las víctimas de un sismo o a los héroes del mismo, ya que el contenido que una persona evoca en situación de entrevista y la forma en que lo hace están mediados por lo que se espera de ella (deseabilidad social); como han reportado los teóricos de la entrevista autobiográfica ${ }^{16}$ (Arfuch, 1995; Grele, 1989).

No es de extrañar que lo anterior suceda si tomamos en cuenta las características del ya mencionado SCE (Michaelian, 2019), especialmente el hecho de que su función principal no sea recordar, sino permitirnos generar simulaciones de situaciones posibles

16 Lo anterior concuerda también con lo señalado por Conway (2005), quien sostiene que la evocación de un recuerdo está mediada por el propósito que la persona tiene al momento de recordar. 
para calcular las consecuencias de una acción (Schacter, 2018). La simulación episódica que se genera en la evocación autobiográfica se orienta por un objetivo, de la misma forma en que una simulación prospectiva o de otro tipo lo harían, pues esa es la función de la simulación en general. Se recuerda para algo, con un propósito; en este caso, a saber, para expresar un significado a través del lenguaje.

No se debe suponer, sin embargo, que el objetivo comunicativo de la persona que evoca necesariamente coincida en todo momento con el del entrevistador. La entrevista, como espacio dialógico, está sujeta a una tensión discursiva donde se debaten dos o más subjetividades (Arfuch, 1995; 2007; Grele, 1989). Los entrevistados a menudo toman rumbos evocativos distintos a los programados y planteados por el entrevistador, construyendo su discurso a partir de lo que consideran relevante para ellos del suceso. Estas "digresiones" del objetivo planteado se dan por muy distintas razones: porque ayuda a los entrevistados a significar un episodio; porque notaron un detalle saliente del que desean abundar; porque necesitan justificar sus acciones del pasado; porque quieren expresar una valoración, ampliación o apología del suceso; porque desean abundar en información que les ayude a construir el recuerdo; porque requieren detenerse a argumentar un punto polémico, una opinión sobre el pasado o hasta una defensa de su testimonio; etc.

\section{B) Idioma de comunicación:}

La activación de las huellas de memoria parece estar relacionada con el idioma en que se realiza la entrevista. La generación y expresión del recuerdo, al elaborarse de forma lingüística, son influenciadas también por este factor.

El lenguaje oral es quizás uno de los factores más importantes que influyen sobre la evocación autobiográfica en la entrevista, no solamente por la interacción dialógica en que esta sucede, sino porque la construcción misma del recuerdo se realiza haciendo uso de él. ${ }^{17}$ Una de las características susceptibles de analizar en la entrevista autobiográfica es el idioma en el que se da la interacción dialógica y la forma en que este podría influir en el proceso de evocación. Dado que una parte importante de las entrevistas autobiográficas del siglo XX (cuando esta metodología cobró auge) se hicieron a inmigrantes, refugiados o grupos indígenas minoritarios, la cuestión del idioma ha sido importante para la entrevista autobiográfica como metodología de la historia oral y la memoria histórica. Para esta última ha tenido especial relevancia, dado que su nacimiento y consolidación como disciplina de las ciencias sociales se dio a partir de la recuperación de discursos autobiográficos de expatriados sobrevivientes

17 Determinar relación entre lenguaje y pensamiento, o más específicamente la influencia de uno sobre otro, es una discusión muy abundante (puede revisarse Chomsky, 2006; Gomila, 2012; Smith \& Kosslyn, 2008; Whorf, 1956), y ciertamente, no es motivo de este artículo. 
de las guerras mundiales, entrevistas que, muchas veces, se han realizado en un idioma distinto a la lengua materna de los entrevistados.

Distintos estudios con personas bilingües (Conway, 2003; Javier, Barroso \& Muñoz, 1993; Marian y Neisser, 2000) han determinado que el idioma influye fuertemente sobre la memoria autobiográfica, mostrando que existe un cambio en los patrones de evocación dependiendo del idioma que las personas emplean para elaborar un recuerdo; es decir, que el discurso autobiográfico y los recuerdos evocados son distintos cuando las personas bilingües evocan empleando cada una de las lenguas que hablan. También se ha detectado que las personas interpretan y codifican de distinta forma una experiencia dependiendo del idioma en que están interactuando (Boroditsky, Ham \& Ramscar, 2002). Esta influencia parece estar presente incluso en la forma en que se expresan identitariamente las personas bilingües que tienen una doble procedencia cultural, pues construyen diferentes discursos de su yo, dependiendo del idioma que estén empleando para hacerlo (Ross, Xun \& Wilson, 1992).

Lo anterior sugiere que nuestros recuerdos autobiográficos están fuertemente ligados al lenguaje (en general) y al idioma (en particular) ${ }^{18}$ que empleamos para recordar una experiencia, lo que nos permite suponer que la activación de las huellas de memoria es susceptible a esta influencia. Al parecer, el idioma podría influir en el tipo de recuerdos que son activados para crear la simulación mnémica, estableciéndose una distinción dependiente del idioma al que un recuerdo está asociado. Por ejemplo, en la investigación aplicada con bilingües (Guttfreund, 1990; Marcos, 1976), se encontró que la psicoterapia puede ser más efectiva cuando la lengua de evocación coincide con aquel en que se vivió la experiencia, o que es posible evocar un mayor número de recuerdos cuando una persona lo hace en su primer idioma (Mortensen, Berntsen \& Bohn, 2015). Se intuye que, dado que la activación de las huellas de memoria habilita el contenido con el que se genera la simulación, el idioma en el que se está interactuando supone la activación de patrones que entre dos idiomas pueden ser similares o concomitantes, pero distintos, lo que se ve reflejado en la construcción del recuerdo.

El efecto anterior permite analizar también la relación tan estrecha que existe entre el idioma y las emociones. Estudios con bilingües, como los anteriormente citados, muestran que existe una diferencia significativa en la intensidad de las emociones experimentadas por los sujetos cuando interactúan en uno u otro idioma, al realizar tareas que implican el uso de memoria autobiográfica (Guttfreund, 1990; Javier, Barroso \& Muñoz, 1993; Marcos, 1976). Es altamente probable que esta diferencia influya también en la construcción del recuerdo, pues la interacción en cierta lengua permite a los sujetos tener una vivencia emocional más intensa, enriqueciendo sensorialmente

18 Es importante puntualizar la distinción entre lenguaje e idioma en este trabajo, entendiendo el primero como la facultad comunicativa humana y al segundo como lengua de un grupo humano. 
la simulación episódica e incrementando la sensación de re experimentación del evento o viaje mental en el tiempo (Lolich \& Azzollini, 2017; Tulving, 2002). Como podemos ver, a diferencia del objetivo comunicativo, el idioma no se relaciona directamente con los estímulos exteroceptivos que influencian la evocación, sino que influye sobre la información interoceptiva que se produce a partir de la experiencia emocional. Los estímulos de este tipo que el sujeto percibe, es decir, la información perceptual interoceptiva que influye sobre la construcción del recuerdo, tiene una intensidad (arousal) -y posiblemente una valencia- diferente en cada lengua de interacción.

Esta dependencia del idioma, por lo visto, se daría no solamente en la activación selectiva de cierto contenido en las huellas de memoria, sino también en la generación de la simulación misma, que se realiza con el contenido activado por ellas. Se ha encontrado que las personas hacen una organización lingüística distinta de sus recuerdos autobiográficos dependiendo del idioma en que se estén expresando (Javier, Barroso \& Muñoz, 1993), variando la cantidad de detalles, experiencias relacionadas y tipo de recuerdos evocados en cada caso. Al parecer, el idioma influye fuertemente sobre el tipo de construcción del recuerdo que se hace al permitir el uso de distintos recursos lingüísticos, dependiendo de la lengua en que se esté interactuando y del dominio que se tenga de ella: estructuras gramaticales, percepción del tiempo o el espacio, empleo del género gramatical, riqueza léxica, uso de jerga, entre muchos otros.

\section{C) Interacción emocional:}

Existe una interacción emocional del entrevistado con el entrevistador, la cual permite que se lleve a cabo un flujo de expresión y retroalimentación no verbal que orienta el discurso.

Una de las características de la evocación en contexto de entrevista, como ya se ha mencionado, es que la misma se realiza en interrelación con otro, lo que supone que la elaboración y expresión del discurso autobiográfico obtiene constantemente retroalimentación por parte del entrevistador. Este último pide a menudo aclaraciones, ampliaciones, detalles o conclusiones sobre el suceso evocado; afirma con palabras, gestos o movimientos cuando comprende lo que se está narrando; reorienta la dirección lógica y afectiva de la evocación (Ortí, 1993), convirtiendo el discurso en el producto de una actividad conjunta (una "negociación”, como le llama De Garay-Arellano, 1999). Todo ello provoca una respuesta en el entrevistado, quien adapta su narración como respuesta ante tal comportamiento.

La imaginación del recuerdo está influenciada por la interacción social, pero esa interacción no solamente se da a través del lenguaje verbal, sino también de las expresiones emocionales de los participantes en la conversación, las cuales constituyen otra forma de comunicación. En este caso, la percepción opera de forma similar a como sucede con el primero de los factores mencionados: a través de la captación de estímulos 
exteroceptivos que el sujeto decodifica para modular su actuación en el contexto. Estos estímulos le proporcionan a la persona que recuerda información no verbal19 sobre la forma en que el entrevistador, los escuchas u otros participantes reciben su relato y las emociones que les causa. En la literatura de la metodología de la entrevista autobiográfica en historia oral (De Garay-Arellano, 1999; González-Monteagudo, 2010; Hinojosa, 2013), a menudo se menciona la importancia del rapport o la sintonía emocional que debe darse entre los interlocutores para lograr un "contrato de confianza" (Arfuch, 2007) y un contexto seguro que permita el libre flujo de los recuerdos. Este es considerado uno de los pilares técnicos de la entrevista (Hinojosa Luján, 2013) y debe ser propiciado por el entrevistador, quien tiene que procurar fomentarlo y sostenerlo a lo largo de la dinámica. Más que verbal, el rapport es una comunicación a través de los gestos, actitudes, posturas, entonaciones y otros elementos de la comunicación que obedecen a una interpretación pragmática. Podemos caracterizarlo como un diálogo que apela a nuestra capacidad para leer las emociones en el otro, sentirnos en confianza y actuar en consecuencia.

La literatura científica sobre nuestra percepción emocional y capacidad de interpretar las emociones de los otros es muy amplia ${ }^{20}$ (y su revisión exhaustiva no es motivo de este trabajo). En cualquier caso, estas investigaciones han revelado que constantemente percibimos e interpretamos emociones ajenas (aunque no siempre de forma correcta). Tal percepción se realiza de forma tan sofisticada que no solo somos capaces de interpretar una emoción a partir de los gestos del rostro de una persona, sino también de incorporar detalles del contexto para realizar una correcta lectura sobre la emoción que alguien experimenta o expresa, como rasgos de las escenas visuales, tipos de voz, orientación cultural, entre otros (Feldman, Mesquita \& Gendron, 2011). Esta habilidad nos ayuda a gestionar las interacciones con otros, al permitirnos planear, modular y evaluar nuestra acción en contextos sociales. Al igual que con el idioma hablado, la interpretación de las emociones del interlocutor proporciona al entrevistado información que retroalimenta su tarea, en este caso, imaginar su recuerdo. La "lectura" de emociones funciona, entonces, como comunicación no verbal que invita a reforzar, resaltar, aclarar, enfatizar, corregir o reorientar el discurso.

\section{Conclusiones y consideraciones finales}

El carácter creativo del recuerdo, que se presenta de forma tan palpable en las evocaciones autobiográficas, ha sido un fenómeno muy discutido por exhibir una de

19 Existe controversia sobre si la percepción emocional se realiza de forma separada (modular) de procesos conceptuales como el lenguaje, aunque recientemente se ha sugerido también que el lenguaje puede servir precisamente como un contexto para la percepción emocional (Feldman, Lindquist \& Gendron, 2007).

20 Como ejemplos pueden revisarse Atkinson \& Adolphs (2011); Keltner \& Ekman (2000); Smith \& Kosslyn (2008); Tsao \& Livingstone (2008). 
las características más importantes, pero también más problemáticas de la memoria humana: su carácter constructivo. Esta capacidad de incorporar elementos novedosos a los recuerdos al momento de evocar ha sido considerada en muchas ocasiones un fallo, debido principalmente a que distintos pensadores y científicos de la historia han empleado, para describir los procesos mnémicos, metáforas que comparan la memoria humana con dispositivos de registro, grabación o almacenamiento, caracterizando el recuerdo como una recuperación de información almacenada; ${ }^{21}$ lo que se ve reforzado por la concepción popular de la memoria que se da en el lenguaje ordinario. Las teorías de simulación ayudan a entender de otra forma esa capacidad, permitiéndonos ver tal carácter creativo como una característica inherente a nuestra gran capacidad imaginativa, y resaltando la enorme ventaja evolutiva que supone ser capaces de realizar construcciones episódicas con información habilitada por distintos sistemas de memoria a partir de la experiencia vivida.

Como se puede ver a partir de los factores mencionados en este texto, la memoria episódica -al menos durante el proceso de evocación- está fuertemente influenciada por estímulos aferentes interoceptivos y exteroceptivos, lo que muestra que la relación entre memoria y percepción no se limita solamente al momento que llamamos codificación. Lo anterior nos lleva a cuestionar qué tanta influencia tiene una sobre otra durante otros procesos cognitivos. Si se toma en cuenta, por ejemplo, que el acto mismo de recordar un evento pone a las huellas de memoria asociadas a este en un estado lábil, como indican los estudios neurológicos de reconsolidación neuronal (ver Labrador \& Restrepo-Castro, 2015; Nader \& Hardt, 2009; Schwabe \& Wolf, 2009), y se considera también que durante la evocación (al menos la episódica) la imaginación emplea información perceptual para recrear el episodio, sería factible sugerir que probablemente gran parte de los recuerdos han sido influenciados un número indeterminado de ocasiones por las situaciones que se viven al momento de recordarlos. Es importante pensar, en futuras reflexiones, las posibilidades y consecuencias epistemológicas de que esta influencia.

Por otra parte, analizar la interacción que se lleva a cabo entre memoria y percepción al momento de la evocación permite enfatizar varias consideraciones importantes.

21 Un recuento histórico de las distintas concepciones de la memoria en filosofía - desde la tabla de cera platónica hasta la imagen del pasado wittgensteniana- puede verse en la sección "History of philosophy of memory" del libro The Routledge Hadbook of Philosophy of Memory (Bernecker \& Michaelian, 2017). En la psicología, aunque las investigaciones de Bartlett (1995) apuntaron tempranamente al concepto de memoria como una habilidad constructiva, el paradigma dominante en los estudios cognitivos durante gran parte del siglo XX empleó la metáfora de la computadora para explicar la cognición humana, caracterizando la memoria como un dispositivo de almacenamiento de información y al recuerdo como un acceso para la recuperación de esta (Baddeley, Eysenck \& Anderson, 2020). Aún hoy, que el paradigma computacional ha dado paso a otros enfoques, la terminología dominante para referirse a los procesos mnémicos en esta disciplina continúa empleando palabras ("codificación", "almacenamiento", "recuperación") que reflejan la herencia de tal metáfora (véase Baddeley, 1990; Smith \& Kosslyn, 2008). 
Primera, la permeabilidad que existe entre procesos cognitivos, ${ }^{22}$ lo cuales no se llevan a cabo de forma aislada, sino que son influenciados unos por otros en una interacción dinámica que permite en cada momento la adaptación del agente al contexto. En segunda, muestra que procesos cognitivos tradicionalmente considerados online como la percepción y offline como la memoria (quizás no haya otro más representativo), en realidad operan de forma simultánea. Un último punto para discutir en futuros trabajos es la relación entre la capacidad humana de efectuar simulaciones episódicas y la habilidad para estructurar de forma narrativa los recuerdos: ¿es constitutiva o causal? Si es esta última, ¿cuál de ellas causa la otra? ¿Qué relación existe entre el desarrollo de los circuitos neuronales que son reclutados para el funcionamiento del SME y el desarrollo como seres narrativos?

Como se puede ver, existen algunos aspectos que han dominado el curso de la argumentación y exploración en este texto. El primero de ellos, es la defensa de la memoria humana como una habilidad cuyo propósito adaptativo y práctico no es el almacenamiento de información. En las entrevistas autobiográficas tradicionalmente se ha entendido a la persona entrevistada como una fuente de información episódica y al entrevistador como un investigador que busca el recuerdo en esa fuente ${ }^{23}$ (AronSchnapper y Hanet, 1993). Pero la persona que evoca es un agente que tiene un propósito en la interacción dialógica (comunicar su recuerdo, el significado de este, etc.), lo que refuerza la perspectiva de que el papel de la memoria humana no se limita a volver disponible la información del pasado, sino a permitirnos actuar en el mundo haciendo uso de esa información (Schacter, 2018). Recordar, desde esta perspectiva, obedece a una necesidad de actuar para cumplir un propósito. La profundización en este rasgo brinda también la oportunidad de discutir y plantear posibles explicaciones a otras cuestiones fundamentales sobre la memoria, como el hecho de que sea constructiva (y no acumulativa), dinámica (que se encuentra en constante cambio) u orientada a la acción.

Un segundo rasgo que subyace a este texto, no menos importante que el anterior (y en realidad profundamente ligado a este), es la defensa de la memoria como una habilidad situada, lo que significa que los seres humanos somos agentes cognitivos actuando en un ambiente físico y social, y que nuestros procesos cognitivos se desarrollan, se desempeñan y se perfeccionan en comunicación y retroalimentación con ese ambiente (ver Barsalou, 2008; Fuchs, 2012; Rietveld, Denys \& Van Westen, 2018).

22 Un tema de continuo debate es la pregunta de si existe un encapsulamiento de los procesos perceptivos de bajo nivel (como la percepción) que los protege de la interferencia de las funciones cognitivas de alto nivel (como memoria o lenguaje). Sin embargo, la investigación empírica ha fortalecido cada vez más el planteamiento de que el sistema cognitivo humano posee una arquitectura interactiva donde las funciones de alto nivel pueden influenciar la cognición de bajo nivel (véase Firestone \& Scholl, 2015; Lupyan \& Clark, 2015), lo que se ha conoce como permeabilidad cognitiva.

23 Como señalan los teóricos de la entrevista en historia oral Aron-Schnapper y Hanet (1993), las entrevistas "recogen de manera privilegiada cierto tipo de información en ciertos medios sociales y en ciertas situaciones [...] se emplean con el propósito de conservar y transmitir las historias [...] y, en general, todo lo que por su carácter no deja huella o deja pocas" (p. 63). 
Explorar los factores contextuales que influyen el proceso de evocación autobiográfica, permite ver que los sistemas cognitivos humanos interactúan con el medio natural y social de forma constante; y que distintos elementos de esos medios afectan -y posiblemente forman parte constitutiva de- procesos cognitivos como la evocación.

Por último, es necesario mencionar que el presente artículo busca inscribirse en la discusión sobre estudios de memoria humana como una aportación teórica que reflexiona sobre un proceso complejo y multidimensional, como es la evocación. Si bien la ambición que lo motiva ha sido describir los procesos cognitivos que esta conlleva y mostrar la relación existente entre percepción y memoria durante su ejecución, el trabajo que se ha llevado a cabo pertenece a la esfera de la reflexión teórica y muchas de las afirmaciones que se expresan, a pesar de estar fundamentadas y referenciadas en trabajos de orden experimental, precisan de un sustento empírico mayor para ser contrastadas y verificadas. Sirva este trabajo, pues, de plataforma y punto de partida para futuras investigaciones interdisciplinarias que permitan ampliar el fundamento empírico de las ideas aquí ensayadas.

\section{Referencias}

Actis, N., Aldini, C., Gardella, L., Lewin, M. \& Tokar, E. (2006). Ese infierno. Conversaciones de cinco mujeres sobrevivientes de la ESMA. Altamira.

Arfuch, L. (1995). La entrevista, una invención dialógica. Paidos.

Arfuch, L. (2005). Problemáticas de la identidad. En L. Arfuch (Comp.), Identidades, sujetos y subjetividades (pp. 21-44). Prometeo Libros.

Arfuch, L. (2007). El espacio biográfico. Dilemas de la subjetividad contemporánea. FCE.

Aron-Schnapper, D. \& Hanet, D. (1993). De Herodoto a la grabadora: fuentes y archivos orales. En J. Aceves Lozano (Comp.), Historia oral (pp. 60-82). Inst. Dr. José María Mora.

Atkinson, A. \& Adolphs, R. (2011). The neuropsychology of face perception: beyond simple dissociations and functional selectivity. Philosophical Transactions of the Royal Society B, 366, 1726-1738. https://doi.org/10.1098/rstb.2010.0349

Barela, L., Miguez, M. \& García, L. (2009). Algunos apuntes sobre Historia oral y cómo abordarla. Dirección General Patrimonio e Instituto Histórico.

Baddeley, A. (1990). Human memory: theory and practice. Allyn \& Bacon.

Baddeley, A., Eysenck, M. \& Anderson, M. (2020). Memory. Routledge.

Barsalou, L. (2005). Continuity of the conceptual system across species. Trends in Cognitive Sciences, 9(7), 309-11. https://doi.org/10.1016/j.tics.2005.05.003

Barsalou, L. (2008). Grounded cognition. Annual Review of Psychology, 59, 617-45. https://doi. org/10.1146/annurev.psych.59.103006.093639 
Boroditsky, L., Ham, W. \& Ramscar, M. (2002). What is universal in event perception? Comparing English \& Indonesian speakers. En W. Gray \& C. Schunn (Eds.), Proceedings of the twenty-fourth annual conference of the Cognitive Science Society (pp. 136-141). https://doi.org/10.4324/9781315782379-61

Chomsky, N. (2006). Language and mind. Cambridge University Press.

Conway, M. (2001). Sensory-perceptual episodic memory and its context: autobiographical memory. Philosophical Transactions of the Royal Society B, 356, 1375-1384. https://doi.org/ 10.1098/rstb.2001.0940

Conway, M. (2003). Cognitive-affective mechanisms and processes in autobiographical memory: Commentary. Memory, 11, 217-224. https://doi.org/10.1080/741938205

Conway, M. (2005). Memory and the self. Journal of Memory and Language, 53(4), 594-628. https://doi.org/10.1016/j.jml.2005.08.005

Cueto, S. (2008). Nacimos en su lucha, viven en la nuestra. Identidad, justicia y memoria en la agrupación HIJOS-La Plata. Trabajo final de grado. Universidad Nacional de La Plata. Facultad de Humanidades y Ciencias de la Educación. http://www.memoria.fahce.unlp.edu.ar/tesis/te.426/te.426.pdf

De Brigard, F. (2014). Is memory for remembering? Recollection as a form of episodic hypothetical thinking. Synthese, 191, 155-185. https://doi.org/10.1111/phc3.12133

Deese, J. (1959). On the prediction of occurrence of particular verbal intrusions in immediate recall. Journal of Experimental Psychology, 58(1), 17-22. https://doi.org/10.1037/h0046671

De Garay-Arellano, G. (1999). La entrevista de historia oral: ¿monólogo o conversación? REDIE. Revista Electrónica de Investigación Educativa, 1(1), 81-89. http://www.redalyc.org/pdf/155/15501107.pdf

De Garay-Arellano, G. (2001). Arquitectura de primera contra ciudadanía de segunda. Ponencia presentada en el XIII International Congress of the Latin American Studies Association. Washington DC, Septiembre 6-8.

Feldman, L., Mesquita, B. \& Gendron, M. (2011). Context in emotion perception. Current Directions in Psychological Science, 20(5), 286-290. https://doi.org/10.1177/0963721411422522

Fisher, J. (2006). Does simulation theory really involve simulation? Philosophical Psychology, 19(4), 417-432. https://doi.org/10.1080/09515080600726377

Firestone, C. \& Scholl, B. (2015). Cognition does not affect perception: Evaluating the evidence for "top-down" effects. The Behavioral and Brain Sciences, 39, e229. https://doi.org/10.1017/S0140525X15000965

Fuchs, T. (2012). The phenomenology of body memory. En S. Koch, T. Fuchs, M. Summa \& C. Muller (Eds.), Body Memory, Metaphor and Movement (pp. 9-23). John Benjamins. https://doi.org/10.1075/aicr.84.03fuc 
González-Monteagudo, J. (2010). La entrevista en Historia oral e Historias de vida: Teoría, método y subjetividad. En L. Benadiba (Ed.), Historial oral: fundamentos metodológicos para reconstruir el pasado desde la diversidad (pp. 21-38). Sur América Ediciones.

Gomila, A. (2012). Verbal minds. Elsevier. https://doi.org/10.1016/C2010-0-67019-2

Guerrero, C., Meilán, J., Carpi, A. \& Palmero, F. (2008). Emoción y memoria: influencia del estado afectivo en el proceso de reconocimiento de palabras. En I. Etxebarría, A. Aritzeta, E. Barbera, M. Choliz, M. P. Jiménez, F. Martínez, P. Mateos \& D. Páez (Eds.), Emoción y motivación: contribuciones actuales (pp. 109-118). Mitxelena

Guerrero Velásquez, C. A. (2020). Factores contextuales que influyen en la evocación autobiográfica en un contexto de entrevista [Tesis de maestría, Universidad Autónoma del estado de Morelos]. http://riaa.uaem.mx/handle/20.500.12055/1510

Grele, R. (1991). La Historia y sus lenguajes en la entrevista de historia oral: Quién contesta a las preguntas de quién y por qué. Historia y Fuente Oral, 5, 111-129. http://www.jstor.org/stable/27753314

Guttfreund, D. (1990). Effects of language usage on the emotional experience of SpanishEnglish and English-Spanish bilinguals. Journal of Consulting and Clinical Psychology, 58, 604-607. https://doi.org/10.1037/0022-006X.58.5.604

Hinojosa Luján, R. (2013). La historia oral y sus aportaciones a la investigación educativa. Revista de Investigación Educativa de la REDIECH, 3(5), 57-65. http://www.redalyc.org/articulo.oa?id=5216/521652343007

Javier, R., Barroso, F. \& Muñoz, M. (1993). Autobiographical memory in bilinguals. Journal of Psycholinguistic Research, 22(3), 319-338. https://doi.org/10.1007/BF01068015

Keltner, D. \& Ekman, P. (2000). Facial expression of emotion. En M. Lewis \& J. Haviland-Jones (Eds.), Handbook of Emotions (pp. 236-249). Guilford Publications, Inc.

Lupyan, G. \& Clark, A. (2015). Words and the world: Predictive coding and the languageperceptioncognition interface. Current Directions in Psychological Science, 24, 279-284. https://doi.org/10.1177/0963721415570732

Marcos, L. (1976). Bilinguals in psychotherapy: Language as an emotional barrier. American Journal of Psychotherapy, 30, 552-560. https://doi.org/10.1176/appi.psychotherapy.1976.30.4.552

Marian, V. \& Neisser, U. (2000). Language-dependent recall of autobiographical memories. Journal of Experimental Psychology: General, 129(3), 361-368. https://doi.org/10.1037//0096-3445.129.3.361

Michaelian, K. (2016). Mental time travel: episodic memory and our knoledge of the personal past. The MIT Press.

Michaelian, K. \& Robins, S. (2018). Beyond the causal theory? Fifty years after Martin and Deutscher (13-32). En K. Michaelian, D. Debus \& D. Perrin (Eds.), New Directions in the Philosophy of Memory (pp. 13-32). Routledge. 
Michaelian, K. \& Sant'Anna, A. (2019). Memory without content? Radical enactivism and (post) causal theories of memory. Synthese. https://doi.org/10.1007/s11229-019-02119-7

Mortensen, L., Berntsen, D. \& Bohn, O. (2015). Retrieval of bilingual autobiographical memories: effects of cue language and cue imageability. Memory, 23(2), 138-156. https://doi.org/10.1080/09658211.2013.873809

Ortí, A. (1993): La apertura y el enfoque cualitativo o estructural: la entrevista abierta semidirectiva y la discusión de grupos. En M. García Ferrando, F. Alvira \& J. Ibañez (Comps.), El análisis de la realidad social: Métodos y técnicas de investigación (pp. 171-203). Alianza.

Pezdeck, K. (2003). Event memory and autobiographical memory for the events of September 11, 2001. Applied Cognitive Psychology, 17, 1033-1045. https://doi.org/10.1002/acp.984

Pinker, S. (1994). The language instinct. Penguin.

Pinto, C. (2013). Los hijos de los exiliados vuelven a Chile: dilemas y desafíos para la integración memoria e identidad. Tesis de posgrado. Universidad Nacional de La Plata. Facultad de Humanidades y Ciencias de la Educación. http://www.memoria.fahce.unlp.edu.ar/tesis/ te.883/te.883.pdf

Portelli, A. (2017). El uso dela entrevista en la Historia oral. Anuario de la Escuela de Historia, 20, 35-48. http://www.anuariodehistoria.unr.edu.ar/ojs/index.php/Anuario/article/view/205/224

Reese, E. \& Fivush, R. (1993). Parental styles of talking about the past. Developmental Psychology, 29(3), 596-606. https://doi.org/10.1037/0012-1649.29.3.596

Rietveld, E., Denys, D. \& Van Westen, M. (2018). Ecological-enactive cognition as engaging with a field of relevant affordances: the skilled intentionality framework (SIF). En A. Newen, L. De Bruin \& S. Gallagher (Eds.), The Oxford Handbook of 4E Cognition. Oxford. https://doi.org/ 10.1093/oxfordhb/9780198735410.013.3

Robin, R. (1996). Identidad, memoria y relato. La imposible narración de sí mismo. Universidad de Buenos Aires.

Roediger, H. \& Mcdermott, K. (1995). Creating false memories: Remembering words not presented in lists. Journal of Experimental Psychology: Learning, Memory, and Cognition, 21(4), 803-814. https://doi.org/10.1037/0278-7393.21.4.803

Ross, M., Xun, W. Q. E. \& Wilson, A. E. (2002). Language and the bicultural self. Personality and Social Psychology Bulletin, 28, 1040-1050. https://doi.org/10.1177/01461672022811003

Shanton, K. \& Goldman, A. (2010). Simulation theory. WIRES Cogni Sci, 1, 527-538. https://doi.org/10.1002/wcs.33

Schacter, D. (1999). The seven sins of memory. Insights from psychology and cognitive neuroscience. American Psychologist. 54(3), 182-203. https://doi.org/10.1037/0003-066X.54.3.182

Schacter, D. (2002). The seven sins of memory: how the mind forgets and remembers. Houghton, Mifflin and Co. 
Schacter, D. (2018). Implicit memory, constructive memory, and imagining the future: a career perspective. Perspectives on Psychological Science, 14(2), 256-272. https://doi.org/10.1177/1745691618803640

Schacter, D. \& Addis, D. (2007). The cognitive neuroscience of constructive memory, remembering the past and imagining the future. Philosophy Transactions of the Royal Society B, 362, 773-786. https://doi.org/10.1098/rstb.2007.2087

Smith, E. \& Kosslyn, S. (2008). Procesos cognitivos: modelos y bases neurales (M. Ramos Platón, Trad.). Pearson Educación.

Szpunar, K., Spreng, N \& Shacter, D. (2014). A taxonomy of prospection: introducing an organizational framework for future oriented cognition. Procedings of the National Academy of Science of the U S A, 111(52), 18414-18421. https://doi.org/10.1073/pnas.141714 4111

Tsao, D. \& Livingstone, M. (2008). Mechanisms of face perception. Annual Reviews Neuroscience, 31, 411-37. https://doi.org/10.1146/annurev.neuro.30.051606.094238

Tulving, A. \& Craik, F. (2000). The Oxford Handbook of Memory. Oxford University Press.

Tulving, E. (2002). Episodic memory: from mind to brain. Annual Review of Psychology, 53, 1-25. https://doi.org/10.1146/annurev.psych.53.100901.135114

Whorf, B. (1956). Language, thought \& reality. The MIT Press. 


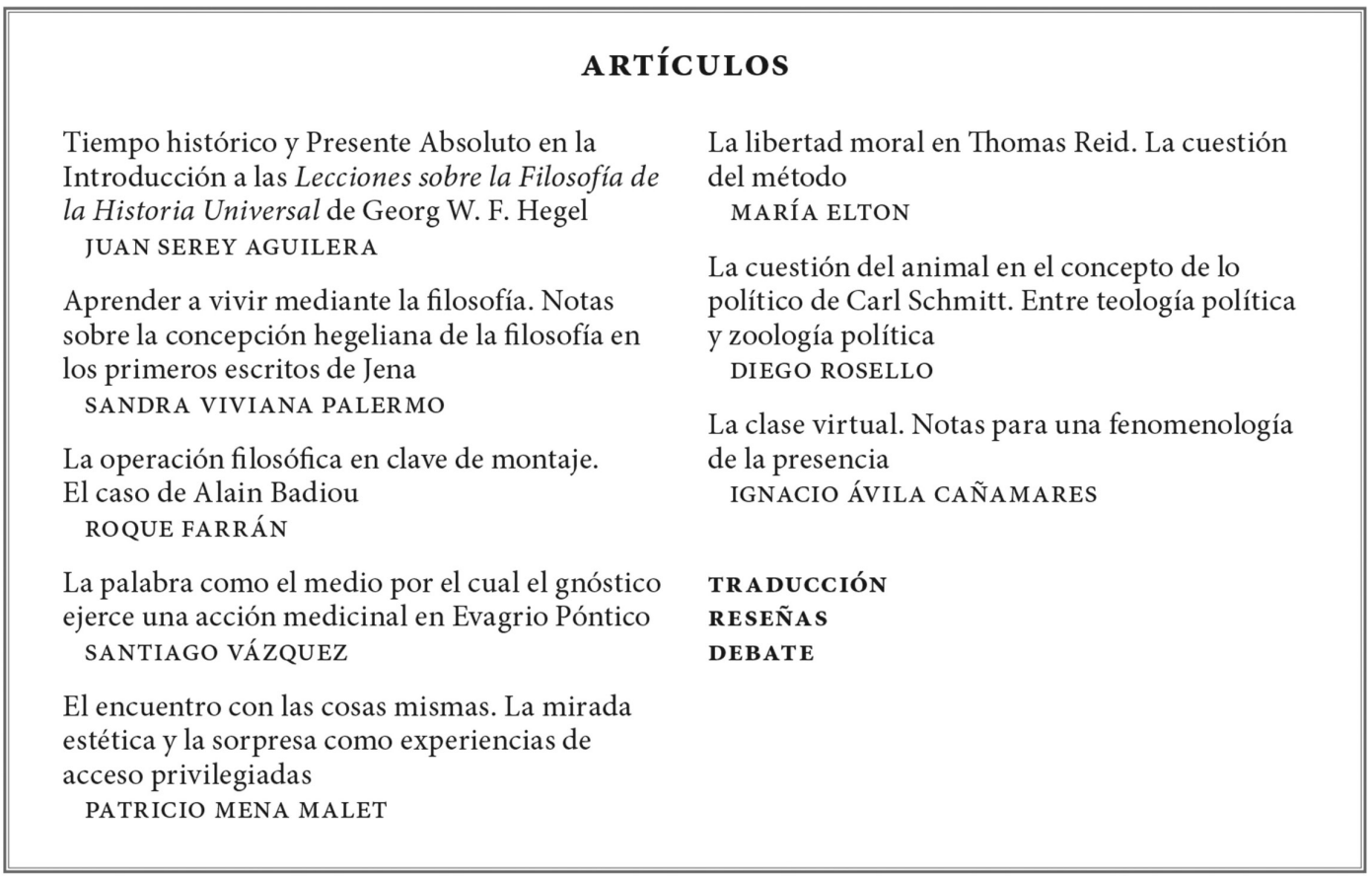

La revista publica tres números al año (enero, mayo y septiembre). Se halla indexada en el IBN-Publindex (Colciencias) y en Philosopher's Index, LATIndex, ULRICH, International Philosophical Bibliography y Philosophical Documentation Center. Además, se encuentra en las siguientes bases de datos:

sCopUs: wWw.scopus.com

CLARIVATE: www.clarivate.com/webofsciencegroup EBSCO: www.ebscohost.com

SCIELO COLOMBIA: www.scielo.org.co/scielo
REDALYC: redalyc.uaemex.mx

DOAJ: www.doaj.org

DIALNET: dialnet.unirioja.es/

oJs: www.ideasyvalores.unal.edu.co

Lo invitamos a suscribirse a la versión impresa de Ideas y Valores, Revista Colombiana de Filosofía, ingresando a la página web de Siglo del Hombre Editores:

www.siglodelhombre.com

\section{Contacto: \\ Ideas y Valores}

Universidad Nacional de Colombia, Cra 30 n. ${ }^{\circ}$ 45-03, Bogotá, Colombia ed. 224, of. 3044, ideasyvalores@gmail.com

Facultad de Ciencias Humanas

Departamento de Filosofía

Sede Bogotá 